\title{
Estimation of Cosmic Induced Contamination in Ultra-low Background Detector Materials
}
E Aguayo
TJ Berguson ${ }^{1}$
RT Kouzes
A Greene ${ }^{2}$
JL Orrell

August 2012

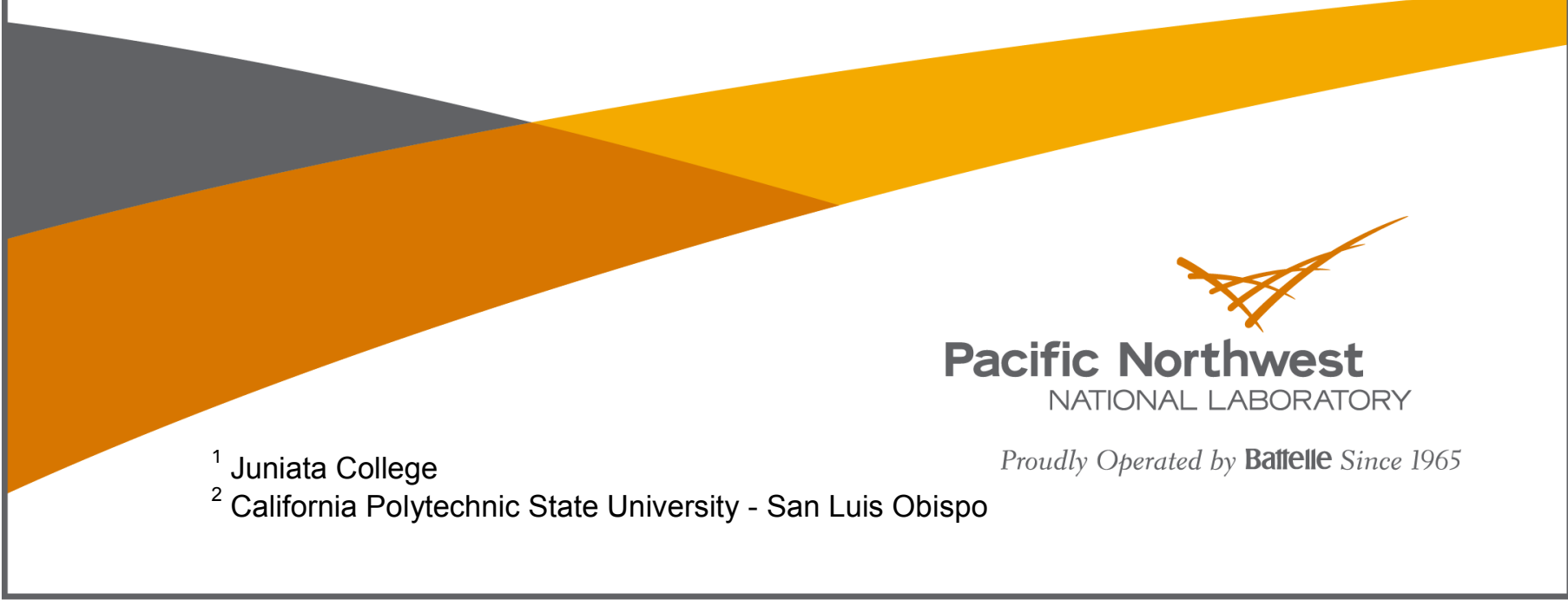




\title{
DISCLAIMER
}

This report was prepared as an account of work sponsored by an agency of the United States Government. Neither the United States Government nor any agency thereof, nor Battelle Memorial Institute, nor any of their employees, makes any warranty, express or implied, or assumes any legal liability or responsibility for the accuracy, completeness, or usefulness of any information, apparatus, product, or process disclosed, or represents that its use would not infringe privately owned rights. Reference herein to any specific commercial product, process, or service by trade name, trademark, manufacturer, or otherwise does not necessarily constitute or imply its endorsement, recommendation, or favoring by the United States Government or any agency thereof, or Battelle Memorial Institute. The views and opinions of authors expressed herein do not necessarily state or reflect those of the United States Government or any agency thereof.

\author{
PACIFIC NORTHWEST NATIONAL LABORATORY \\ operated by \\ BATTELLE \\ for the \\ UNITED STATES DEPARTMENT OF ENERGY \\ under Contract DE-AC05-76RL01830
}

Printed in the United States of America
Available to DOE and DOE contractors from the Office of Scientific and Technical Information,
P.O. Box 62, Oak Ridge, TN 37831-0062;
ph: (865) 576-8401
fax: $(865) 576-5728$
email: reports@adonis.osti.gov

\footnotetext{
Available to the public from the National Technical Information Service, U.S. Department of Commerce, 5285 Port Royal Rd., Springfield, VA 22161 ph: (800) 553-6847 fax: $(703) 605-6900$ email: orders@ntis.fedworld.gov online ordering: http://www.ntis.gov/ordering.htm
}

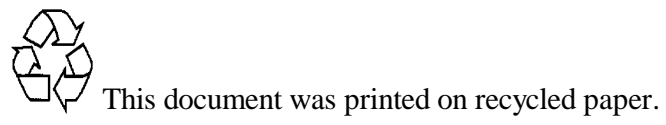




\title{
Estimation of Cosmic Induced Contamination in Ultra-low Background Detector Materials
}

\author{
E Aguayo \\ RT Kouzes \\ TJ Berguson ${ }^{1}$ \\ JL Orrell \\ A Greene ${ }^{2}$
}

August 2012

Prepared for

the U.S. Department of Energy

under Contract DE-AC05-76RL01830

Pacific Northwest National Laboratory

Richland, Washington 99352

\footnotetext{
1 Juniata College

${ }^{2}$ California Polytechnic State University - San Luis Obispo
} 



\section{Executive Summary}

This document presents the result of investigating a way to reliably determine cosmic induced backgrounds for ultra-low background materials. In particular, it focuses on those radioisotopes produced by the interactions with cosmic ray particles in the detector materials that act as a background for experiments looking for neutrinoless double beta decay. This investigation is motivated by the desire to determine background contributions from cosmic ray activation of the electroformed copper that is being used in the construction of the MAJORANA DEMONSTRATOR. The most important radioisotope produced in copper that contributes to the background budget is ${ }^{60} \mathrm{Co}$, which has the potential to deposit energy in the region of interest of this experiment. Cobalt-60 is produced via cosmic ray neutron collisions in the copper. This investigation aims to provide a method for determining whether or not the copper has been exposed to cosmic radiation beyond the threshold which the Majorana Project has established as the maximum exposure. This threshold is set by the Project as the expected contribution of this source of background to the overall background budget.

One way to estimate cosmic ray neutron exposure of materials on the surface of the Earth is to relate it to the cosmic ray muon exposure. Muons are minimum-ionizing particles and the available technologies to detect muons are easier to implement than those to detect neutrons. We present the results of using a portable, ruggedized muon detector, the $\mu$-Witness made by our research group, for determination of muon exposure of materials for the MAJORANA DEMONSTRATOR. From the muon flux measurement, this report presents a method to estimate equivalent sea-level exposure, and then infer the neutron exposure of the tracked material and thus the cosmogenic activation of the copper.

This report combines measurements of the muon flux taken by the $\mu$-Witness detector with Geant 4 simulations in order to assure our understanding of the $\mu$-Witness prototype. As a proof of concept, we present the results of using this detector with electroformed copper during its transport from Pacific Northwest National Laboratory, where the copper is grown, to the underground lab in Lead, South Dakota, where the experiment is being deployed. The development of a code to be used with the Majorana parts tracking database, designed to aid in estimating the cosmogenic activation, is also presented. 



\section{Acronyms and Abbreviations}

$0 v \beta \beta$

Geant4

GERDA

HPGe

MJD

mwe

PNNL

SURF

$\mu$-Witness
Neutrinoless double-beta decay

Geometry and Transport 4

European ${ }^{76} \mathrm{Ge}$ Experiment

High-purity germanium

MAJORANA DEMONSTRATOR

meters water equivalent

Pacific Northwest National Laboratory

Sanford Underground Research Facility

Muon Witness Detector 



\section{Contents}

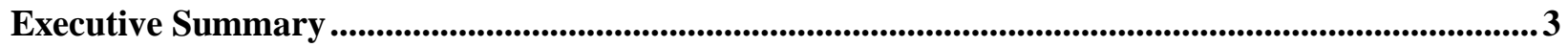

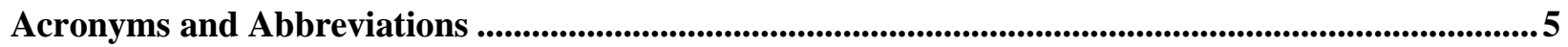

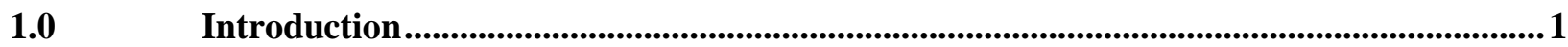

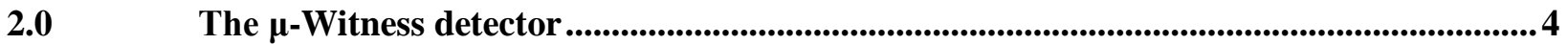

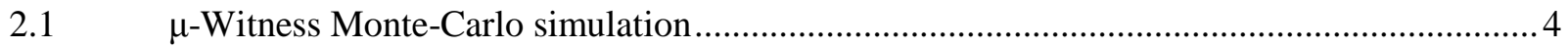

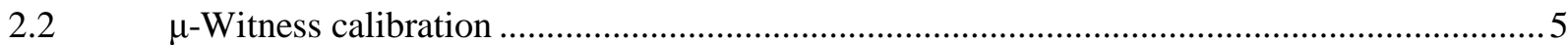

3.0 First experimental result: copper activation estimation during travel to South Dakota.7

3.1 Background rate estimation from equivalent sea-level exposure.......................................... 8

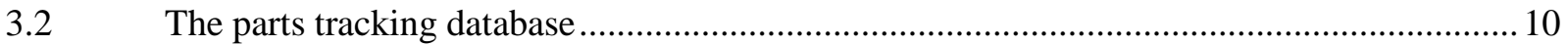

3.3 Background estimation tool for the parts tracking database ............................................... 11

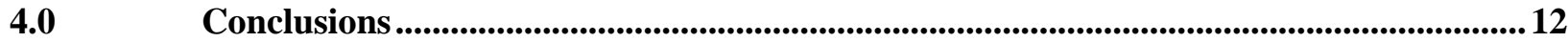

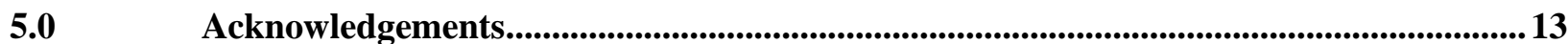

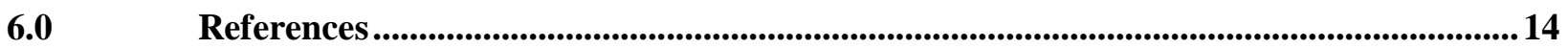

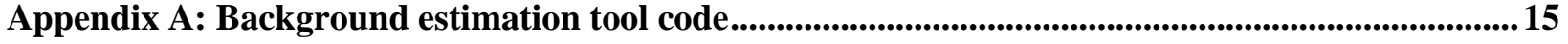




\section{Figures}

Figure 1: A computer rendering of an MJD module (shield not shown)...................................... 1

Figure 2: Electroformed copper part that will become part of the MJD module..............................2

Figure 3: A plot of the Cross Section vs Incident Energy for the production of ${ }^{60} \mathrm{Co}$ from ${ }^{63} \mathrm{Cu}$,

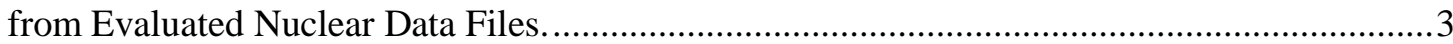

Figure 4: A plot of the $\mu$-Witness count rate vs. mwe; the mwe was estimated based upon a linear

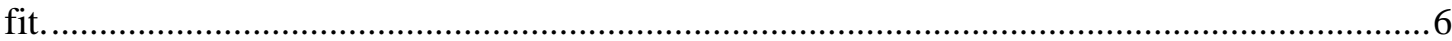

Figure 5: The $\mu$-Witness detector attached to one of the steel drums used to transport the electroformed copper during shipment from PNNL to SURF. ..............................................

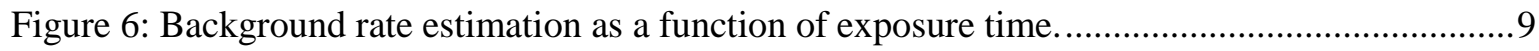

\section{Tables}

Table 1: The efficiencies determined by simulation of the $\mu$-Witness detector and the large panels with 100,000 muons generated.

Table 2: Background rates from cosmogenic produced Co60 allowed for the MJD. (Extracted from MJD background budget [Gehman, 2010]) ................................................................ 8

Table 3: Copper mass of the different parts of the MJD module inside the shield........................... 8

Table 4: Sea-level equivalent exposure of different routes of interest for Majorana.......................11 


\subsection{Introduction}

The question as to whether neutrinos are Dirac or Majorana particles has not yet been answered. The MAJORANA DEMONSTRATOR (MJD) is designed to explore whether an experimental search for neutrinoless double beta decay based on an array of enriched germanium $(\mathrm{Ge})$ detectors inside a copper cryostats has the performance parameters, in particular sufficiently low backgrounds, required to pursue a next generation tonne scale experiment. Figure 1 shows a rendering of one of the MJD modules. The MJD experiment will use two of these modules to reach a total germanium mass of $60 \mathrm{~kg}$ of $\mathrm{Ge}$. The module features ultra-low background building materials for all parts that will be placed inside the radiation shield. In particular, all the parts rendered in orange will be made of electroformed copper. The residual uranium-chain, thorium-chain, and cosmogenic radionuclide concentration levels in this material are a critical factor for the success of the MJD ultimate goal to reach background rates of 3 counts/tonyear (after analysis cuts) in a $4 \mathrm{keV}$ region of interest (ROI) around $2039 \mathrm{keV}$.

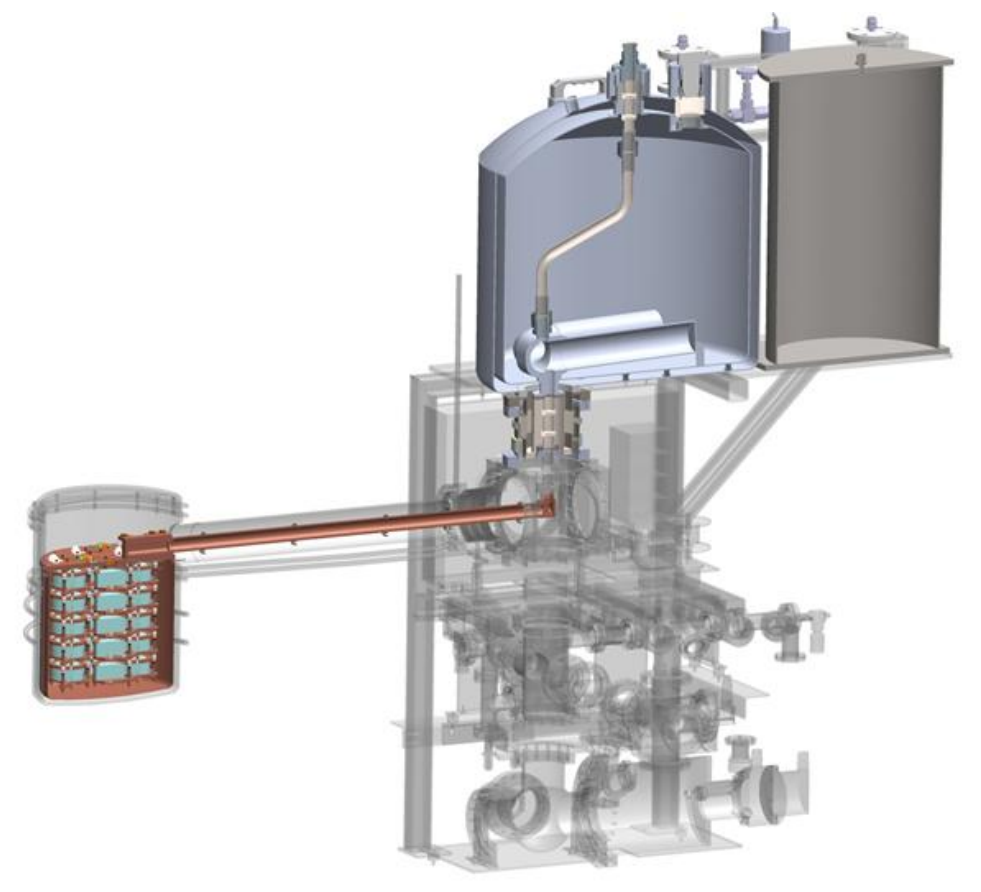

Figure 1: A computer rendering of an MJD module (shield not shown).

Cosmic-ray particles are a direct and immediate background in radiation detection based experiments located on the Earth's surface. To avoid this direct background, experiments are placed in deep underground labs that shield against a significant fraction of the cosmic-ray flux. However, cosmic rays (primarily neutrons and protons) can generate radioisotopes with long half-lives in the materials of the experiment, thus creating a background source from cosmic-ray exposure of parts fabricated on the Earth's surface. These radioisotopes are produced via collisions of the hadronic components of the cosmic ray shower. In particular the hadronic components have an estimated integral flux of $1201 /\left(\mathrm{m}^{2} \cdot \mathrm{s}\right)$ for neutrons, and $3.81 /\left(\mathrm{m}^{2} \cdot \mathrm{s}\right)$ for protons at the Earth's surface at sea-level [Grieder 2001]. The MJD experiment will utilize high-purity germanium (HPGe) detectors housed in electroformed copper cryostats, both of which are susceptible to cosmogenic activation, with product radioisotopes that undergo 
radioactive decay with the potential to deposit energy in the region of interest of the experiment. Figure 2 shows one of the electroformed copper parts of the MJD. This particular part will become the thermosyphon pipe, part of the cryogenic system of the MJD. This part was electroformed at Pacific Northwest National Laboratory (PNNL), so it will undergo cosmogenic activation during handling and transportation while on the Earth's surface. One aspect of the project is to ensure that the activation of this part (and others like it) are below the allowed limits contained in the background budget of the MJD.

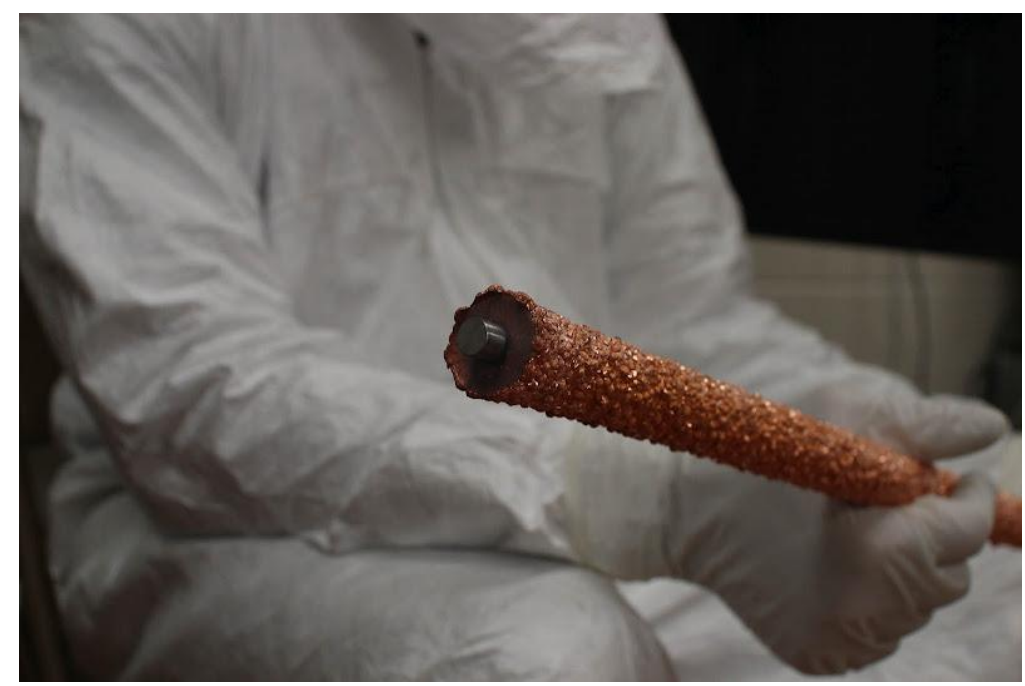

Figure 2: Electroformed copper part that will become part of the MJD module.

Although cosmic-ray particles are capable of forming multiple radionuclides within germanium and copper, the one of greatest interest for this study is ${ }^{60} \mathrm{Co}$, which decays in the energy region of interest for Ge neutrinoless double-beta decay [Laubenstein and Heusser 2009]. For this reason, it is essential to minimize the exposure of the Ge and electroformed copper to cosmic radiation in order to preserve the integrity of future results from the MAJORANA DEMONSTRATOR. This research focuses on estimating the activation of ${ }^{60} \mathrm{Co}$ within electroformed copper to provide an input to the cosmogenic background estimation for the Majorana Project.

Cosmogenic activation is typically caused by direct reactions, spallation or capture; in the case of the activation of copper, the means of production is probably direct reactions plus spallation at higher energies. Cobalt-60 is produced in copper when a neutron reacts with a ${ }^{63} \mathrm{Cu}$ nucleus and emits an alpha particle. A plot of the cross section versus incident energy of this process can be seen in Figure 3, which peaks at approximately $13 \mathrm{MeV}$. Note that the data ends at $20 \mathrm{MeV}$, due to the limited energy rage of generation of neutrons in reactors where this data is acquired. The production rate of ${ }^{60} \mathrm{Co}$ in copper due to cosmogenic activation at sea level was determined to be approximately $86.4 \pm 7.8$ nuclei $(\mathrm{kg} \cdot \mathrm{day})^{-1}$ (about $1 \pm 0.1 \mathrm{mBq} \cdot \mathrm{kg}^{-1}$ ) [Laubenstein and Heusser 2009]. 
PNNL-21696

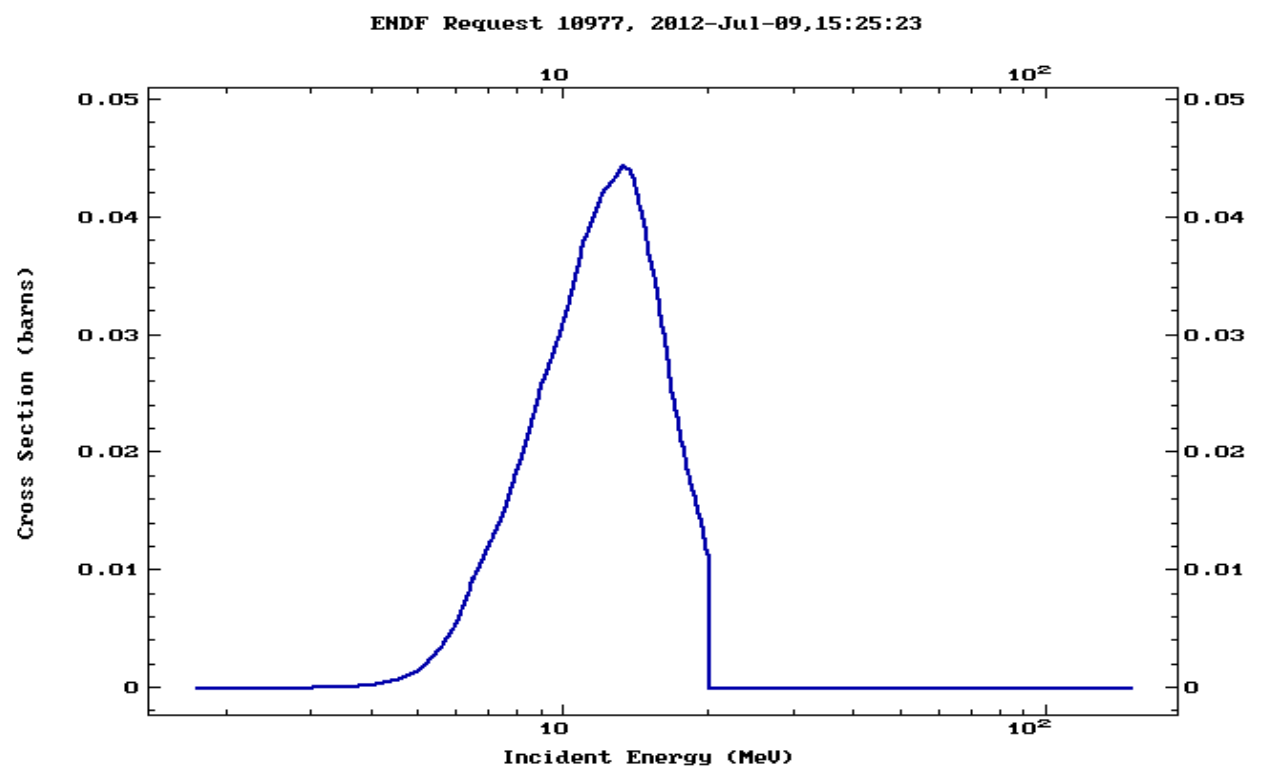

Figure 3: A plot of the Cross Section vs Incident Energy for the production of ${ }^{60} \mathrm{Co}$ from ${ }^{63} \mathrm{Cu}$, from Evaluated Nuclear Data Files. 


\subsection{The $\mu$-Witness detector}

A muon detector, known as the $\mu$-Witness, was designed and built in order to monitor the cosmic ray exposure that materials receive when they are above ground. One key to accurately predicting the sealevel equivalent exposure using the $\mu$-Witness detector was determining the detector's efficiency for muons. The methods of determining the efficiency relied on two methods, direct measurements and simulations. Measurements taken with the $\mu$-Witness detector were compared to simulations of particles through the detector using a simulation tool developed by [Aguayo et al. 2010]. This simulation package includes a Geant4 toolkit, combined with the CRY library [Hagmann, 2008] and ROOT [Brun 1996] data analysis tool.

The $\mu$-Witness detector consists of two $17.5 \times 15 \times 4 \mathrm{~cm}^{3}$ polyvinyl-toluene (PVT) panels set up in electronic coincidence within a ruggedized case $\left(30 \times 20 \times 15 \mathrm{~cm}^{3}\right)$. The two panels are $8 \mathrm{~cm}$ apart, parallel with one above the other, and with a $0.635 \mathrm{~cm}$ lead sheet placed between them to reduce gamma ray and beta ray coincidences. This ruggedized detector was specifically designed to travel alongside materials for the Majorana Project that are susceptible to cosmogenic activation, primarily the electroformed copper and the HPGe materials and detectors.

\section{$2.1 \mu$-Witness Monte-Carlo simulation}

Determining the efficiency of the $\mu$-Witness detector to muons was a major aim for running simulations of the detector with the same dimensions and geometry as the actual $\mu$-Witness in Geant 4 utilizing the CRY library and ROOT analysis tool. A larger muon counter (to reduce systematic errors due to active surface area) was used to compare results with the $\mu$-Witness. The larger muon monitor is composed of two large sensitive area panels $\left(81 \times 92.5 \times 5 \mathrm{~cm}^{3}\right)$. These panels are $4 \mathrm{~cm}$ thick and were placed normal to the azimuthal angle, one on top of the other. There were a total of six simulation runs conducted, each with 100,000 muons generated. The first run was the default setting for Geant 4 , and the next five used seed numbers selected using a random number generator for integers between 0 and 1000 [Wolfram|Alpha 2012]. The efficiencies from the results of these runs can be seen in Table 1.

\begin{tabular}{|c|c|l|l|l|}
\hline $\begin{array}{c}\text { Simulation } \\
\text { Seed }\end{array}$ & $\begin{array}{c}\text { Efficiency } \\
\mu \text {-Witness } \\
\text { (Sea-level) }\end{array}$ & $\begin{array}{c}\text { Efficiency } \\
\text { Large Panels }\end{array}$ & $\begin{array}{c}\text { Efficiency } \mu \text {-Witness Relative to } \\
\text { Large Panels }\end{array}$ & $\begin{array}{c}\text { Efficiency } \mu \text {-Witness } \\
(2100 \text { m elevation })\end{array}$ \\
\hline 121725 & 0.430 & 0.906 & 0.474 & 0.375 \\
\hline 201882 & 0.400 & 0.906 & 0.437 & 0.444 \\
\hline 552269 & 0.431 & 0.906 & 0.476 & 0.427 \\
\hline 651733 & 0.445 & 0.910 & 0.490 & 0.395 \\
\hline 792111 & 0.416 & 0.905 & 0.460 & 0.449 \\
\hline Default & 0.414 & 0.908 & 0.455 & 0.417 \\
\hline Average & $0.422 \pm 0.03$ & $0.907 \pm 0.01$ & $0.465 \pm 0.03$ & $0.418 \pm 0.03$ \\
\hline
\end{tabular}

Table 1: The efficiencies determined by simulation of the $\mu$-Witness detector and the large panels with 100,000 muons generated. 
Muons were produced $0.5 \mathrm{~m}$ away from the top panel, directed with the correct cosmic ray angular distribution, and were generated in an area of a $3 \times 3 \mathrm{~m}^{2}$. The simulations took place at sea level, with the exception of the $\mu$-Witness Elevation simulations, which took place at $2100 \mathrm{~m}$ above sea level. The efficiency was taken to be the efficiency of muons to generate a coincident count, and was thus given by the equation,

$$
\text { Efficiency }=\frac{N_{\text {Coincidence }}}{N_{\text {Total }}}
$$

where $N_{\text {Coincidence }}$ is the number of muons which traveled through both detectors, and $N_{\text {Total }}$ is the total number of muons which went through at least one detector. The $\mu$-Witness Relative to Elevation column lists the change in the efficiency of the $\mu$-Witness going from an elevation $0 \mathrm{~m}$ to $2100 \mathrm{~m}$. This was done in order to determine if the efficiency was dependent upon elevation.

\section{$2.2 \mu$-Witness calibration}

A previous study investigated the accuracy of the $\mu$-Witness detector by comparing direct measurements with the detector to measurements with larger PVT panels stacked on top of each other [Aguayo et al. 2012]. The relative efficiency of the $\mu$-Witness detector was determined to be $60 \pm 3 \%$ in that study. This work looks into the efficiency of the $\mu$-Witness based upon the expected muon flux at PNNL, as well as based upon geometric limitations of the detector due to the separation between the panels and the anisotropic background of muons at sea-level.

The expected muon background on site at PNNL was determined using equations from [Ziegler 1998]. Ziegler presented an approximation,

$$
\frac{I_{1}}{I_{2}}=e^{\left(A_{2}-A_{1}\right) L}
$$

where $I_{1}$ is the flux at altitude $A_{1}$, converted to terms of pressure, $I_{2}$ is the flux at $A_{2}$, and $L$ is the mean attenuation length of muons. New York City was selected as the datum flux in [Ziegler 1998], and so we have selected New York City as a reference as well; Ziegler reports a theoretical calculation of the muon flux in New York City (sea level) to be $207 \mathrm{~m}^{-2} \cdot \mathrm{s}^{-1}$. Using this for $I_{2}, 261 \mathrm{~g} / \mathrm{cm}^{2}$ for L, and calculating $A_{1}$ and $A_{2}$ using the equation,

$$
A=1033-0.03648 H+\left(4.26 \times 10^{-7} H^{2}\right)
$$

where $A$ is in $\mathrm{g} / \mathrm{cm}^{2}$ and $H$ is $0 \mathrm{~m}_{-}$for New York City, and $116 \mathrm{~m}$ for PNNL.

Using $I_{1}$ and $A_{1}$ to correspond to PNNL and $I_{2}$ and $A_{2}$ to correspond to New York City, the muon flux at PNNL was calculated to be 1.04 times greater than that of New York City. The expected muon flux at PNNL was thus determined to be $217 \mathrm{~m}^{-2} \cdot \mathrm{s}^{-1}$. The $\mu$-Witness measurements correspond to a muon background at PNNL of $236 \pm 17.4 \mathrm{~m}^{-2} \cdot \mathrm{s}^{-1}$, after taking the efficiency as determined in simulations into account. This estimate is nearly consistent with the expected background of $217 \mathrm{~m}^{-2} \cdot \mathrm{s}^{-1}$. More work needs to be done in order to determine an associated error with calculations of the expected background flux at specific sites. 
The $\mu$-Witness detector collected a variety of measurements at PNNL, inside, outside, and in the shallow underground lab, which has a depth of $\sim 30$ meters water equivalent (mwe). The $\mu$-Witness count rate was $2.51 \pm 0.04$ muons/s, $2.61 \pm 0.05$ muons/s, and $0.40 \pm 0.01$ muons $/ \mathrm{s}$ inside, outside and in the underground lab, respectively. These rates scale with area to the previously discussed rates. Indoor measurements were expected to be lower than outdoors, as the laboratory overhead serves as overburden, and was estimated to be about 2 mwe, as seen in Figure 4.

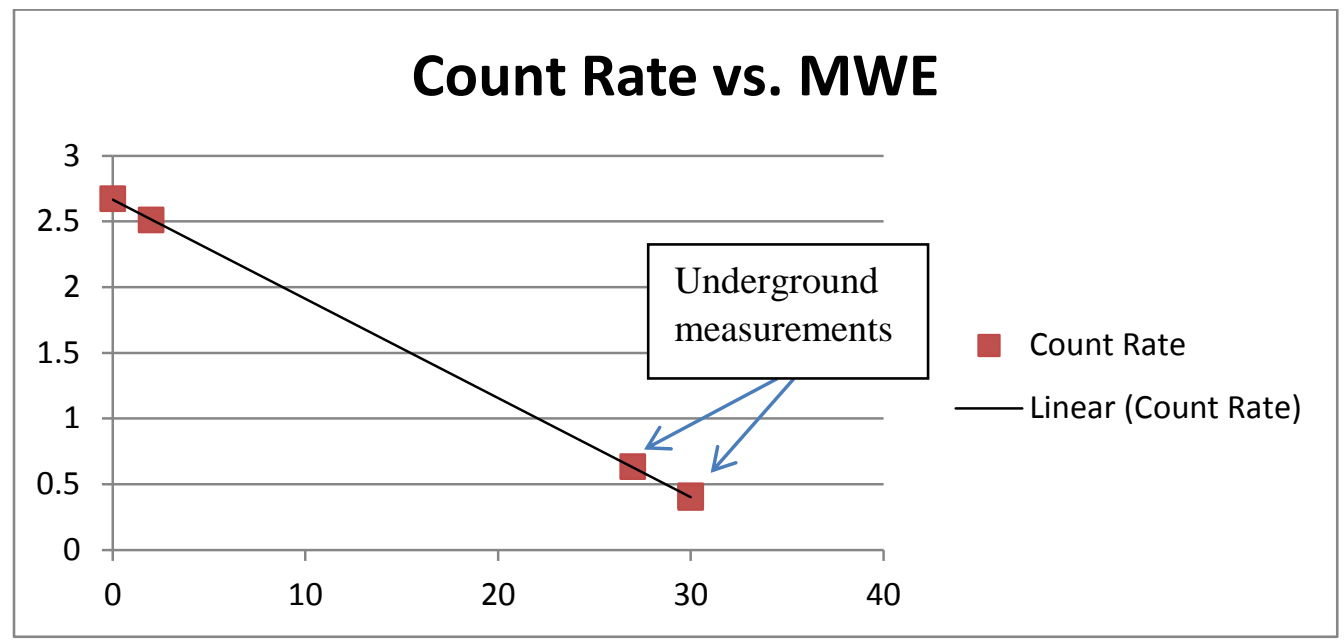

Figure 4: A plot of the $\mu$-Witness count rate vs. mwe; the mwe was estimated based upon a linear fit. 


\subsection{First experimental result: copper activation estimation during travel to South Dakota}

Electroformed copper to be used in the MAJORANA DEMONSTRATOR was shipped from PNNL to the site of the experiment, the Sanford Underground Research Facility (SURF) in Lead, South Dakota in June 2012. The $\mu$-Witness detector was attached to one of the steel drums containing the copper as seen in Figure 5. By doing so, it would be possible to determine the integral count rate of muons during shipment and then calculate the copper's exposure relative to sea level.

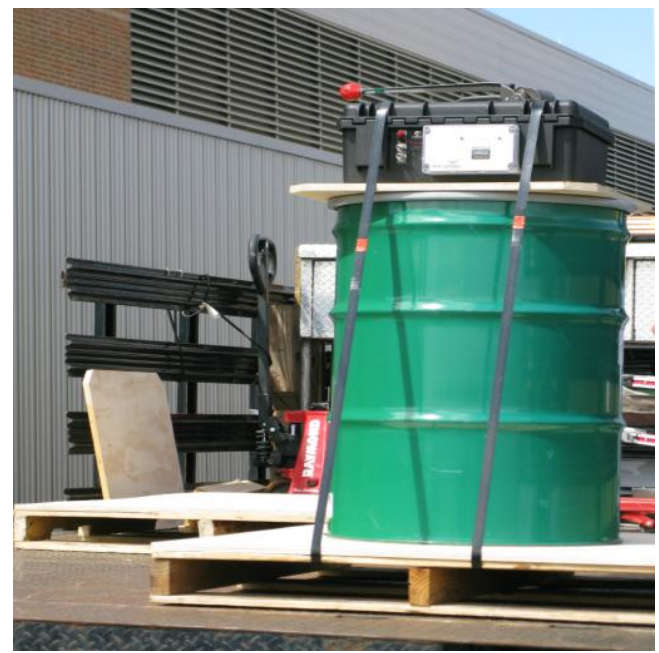

Figure 5: The $\mu$-Witness detector attached to one of the steel drums used to transport the electroformed copper during shipment from PNNL to SURF.

The integral count rate can be used in calculating the sea-level equivalent exposure by using the equation,

Sea Level Equivalent Exposure $=I C R \cdot\left(170 \frac{\mu}{\mathrm{m}^{2} \cdot \mathrm{s}} \cdot 0.02625 \mathrm{~m}^{2} \cdot 86.4 \times 10^{3} \frac{\mathrm{s}}{\mathrm{day}} \cdot \text { Efficiency }\right)^{-1}$

where ICR is the integral count rate, $170 \mu \cdot \mathrm{m}^{-2} \cdot \mathrm{s}^{-1}$ is the approximate muon background at sea level [Grieder 2001], $0.02625 \mathrm{~m}^{2}$ is the area of the detector, and Efficiency is the efficiency of the $\mu$-Witness to muons, which is a focus of this study. 
PNNL-21696

\subsection{Background rate estimation from equivalent sea-level exposure}

The MJD experiment's goal is to demonstrate a background level of no more than 3 counts/ton-year (after analysis cuts) in a $4 \mathrm{keV}$ region of interest (ROI) around $2039 \mathrm{keV}$. When extrapolated to the 1-ton experiment, this corresponds to a background of no more than 1 count/ton-year in the $4 \mathrm{keV}$ ROI. Table 2 shows the allowed background contribution from ${ }^{60} \mathrm{Co}$ from all the copper in the detector to the total background budget. All ROI contributions are in counts per ton per year. Total ROI expected count rate from all sources of background will be 20.6 counts before analysis cuts, which will decrease to 2.9 after analysis cuts. [Gehman, 2010].

\begin{tabular}{|l|l|l|}
\hline Background & Before cuts (c/ROI/t/y) & After cuts (c/ROI/t/y) \\
\hline${ }^{60} \mathrm{Co}$ in $\mathrm{Cu}$ & 0.001716 & 0.0000099 \\
\hline
\end{tabular}

Table 2: Background rates from cosmogenic produced Co60 allowed for the MJD. (Extracted from MJD background budget [Gehman, 2010])

\begin{tabular}{|l|l|}
\hline Component & Mass \\
\hline Detector Mounts & $6.8 \mathrm{~kg}$ \\
\hline Cold plate & $15.6 \mathrm{~kg}$ \\
\hline Cryostat & $124.5 \mathrm{~kg}$ \\
\hline Inner copper shield & $594 \mathrm{~kg}$ \\
\hline
\end{tabular}

Table 3: Copper mass of the different parts of the MJD module inside the shield.

The background rate induced by the cosmogenic ${ }^{60} \mathrm{Co}$ activity (in units of c/ROI/t/y) of the copper can be calculated using the equation [Gehman, 2008]:

$R=\frac{M_{C u}}{M_{G e}} P \frac{\tau}{t_{E x p}}\left(1-e^{-\frac{t_{\text {Surf }}}{\tau}}\right) e^{-\frac{t_{S t a r t}}{\tau}}\left(\epsilon_{E \text { Form }}\right)\left(\epsilon_{R O I \times G r a n}\right)\left(\epsilon_{P S A}\right)\left(1-e^{-\frac{t_{E x p}}{\tau}}\right)$

Where

- $\varepsilon_{\mathrm{ROI}} \times \varepsilon_{\text {Granularity }}=5.8 \times 10^{-6} \mathrm{c} / \mathrm{ROI} /$ decay $\left({ }^{60} \mathrm{Co}\right.$ in supports efficiency from the Demonstrator Backgrounds spreadsheet)

- $\quad \varepsilon_{\text {Electroforming }}=10^{-6}$ (fraction of ${ }^{60} \mathrm{Co}$ remaining in bulk copper after electroforming)

- $\varepsilon_{\mathrm{PSA}}=0.17, \mathrm{e}_{\mathrm{SSTC}}=1.0\left({ }^{60} \mathrm{Co}\right.$ in supports efficiency from the Demonstrator Backgrounds spreadsheet)

- $\mathrm{P}=$ Production rate $=86.4 \pm 7.8(\mathrm{~kg} \cdot \mathrm{day})^{-1}\left(\right.$ about $\left.1 \pm 0.1 \mathrm{mBq} \cdot \mathrm{kg}^{-1}\right)[$ Heusser $1994 \mathrm{and} / \mathrm{or}$ Laubenstein and Heusser 2009].

- $\quad \mathrm{t}_{\text {Surface }}=$ Sea-level equivalent time on surface.

- $\mathrm{M}_{\mathrm{Cu}}=$ Copper mass

- $\mathrm{M}_{\mathrm{Ge}}=0.06$ tonnes (One cryostat) 
- $\mathrm{t}_{\text {Start }}=100$ days (time underground BEFORE the experiment starts--this is essentially a cool-down time)

- $\mathrm{t}_{\mathrm{Exp}}=3$ years

- $\tau={ }^{60}$ Co lifetime $=7.6$ years $\left(\mathrm{T}_{1 / 2}=5.3\right.$ years $)$

Using the previous equation and the different masses for the parts in the MJD module, one can infer the expected background rate from each of the parts as a function of sea level equivalent exposure days. Such a calculation result is presented in Figure 6. This is a worst case scenario, since the efficiencies (Pulse shape analysis, single site time correlation) used to calculate these backgrounds are all the same and these efficiencies were calculated for the detector mount parts, which are the closest parts to the detector mass.

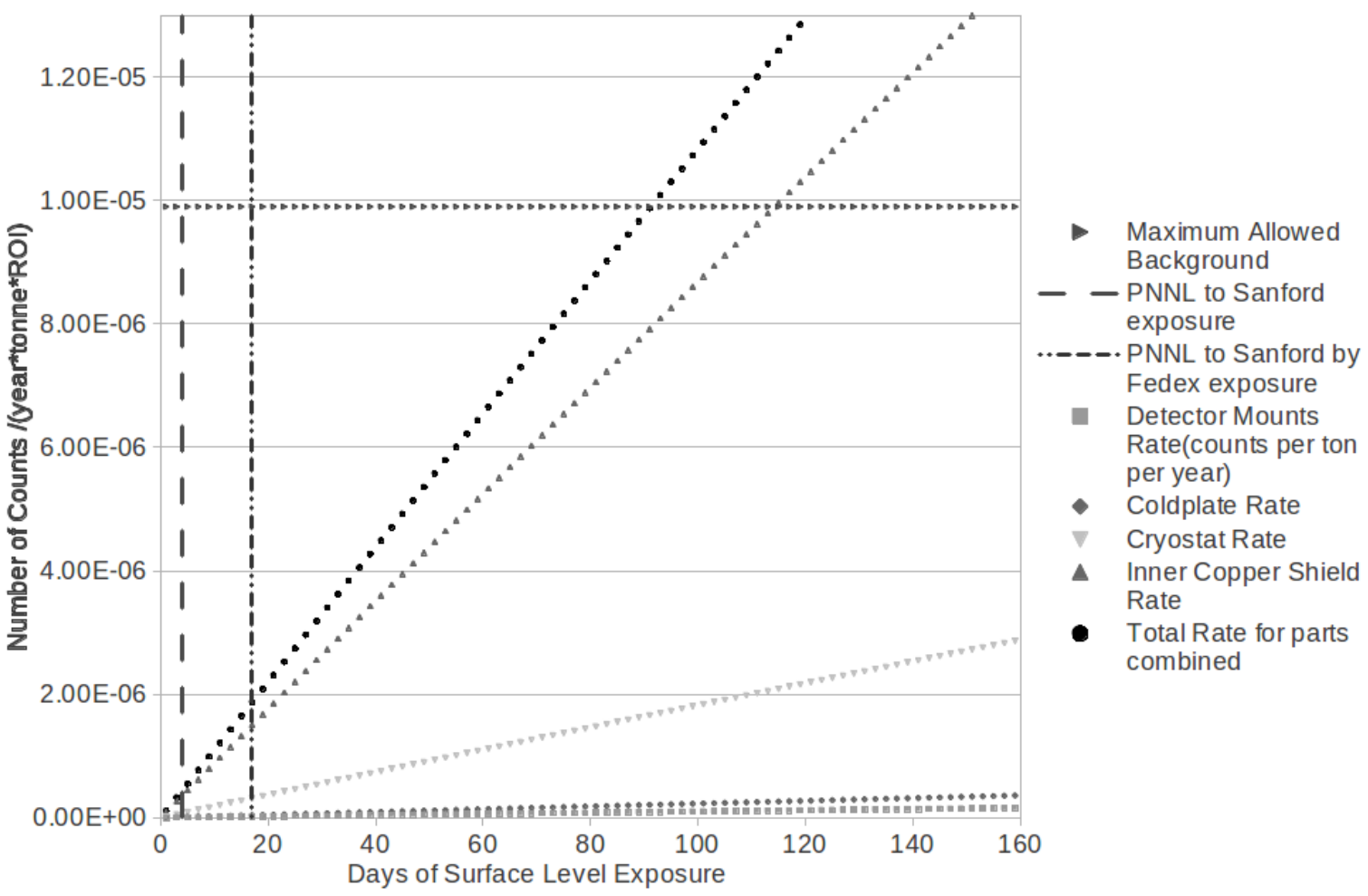

Figure 6: Background rate estimation as a function of exposure time.

The left most vertical dashed line in the figure represents the equivalent exposure time assuming a trip with no delays or stops, straight from PNNL to Lead, SD. The vertical line to the right represents the exposure using actual tracking information for a copper mandrel shipped in June 2012. Notice that the exposure time for the June 2012 shipping packages was up to a factor of 4 higher due to stops and delays (particularly a several day delay in Denver, at very high elevation). The equivalent sea-level exposure is dominated by the time that the material spends at different altitudes. 
PNNL-21696

\subsection{The parts tracking database}

Keeping detailed records of each part that makes up the MJD detector is an essential requisite for calculating each background contribution. This database will store records of every part and its location, route and responsible party, among other information. For this purpose, a database was created by Kyle Snavely (University of North Carolina) that uses CouchDB, a NoSQL framework that uses JSON documents and javascript queries. Data from the database could be accessed in a variety of ways - for convenience and portability, the couchdb4j library for the Java programming language was chosen for this purpose. An example query response from to consult transportation records from the database and parsing their information is given below:

sent from : UNC,North Carolina on 8/17/2011 19:53 arrived at LBNL,California on 8/22/2011 23:12

sent from : UNC,North Carolina on 9/06/2011 14:34 arrived at PNNL, Washington on 09/12/2011

sent from : UNC,North Carolina on 9/08/2011 20:39 arrived at PNNL,Washington on 09/12/2011

Below is an example of a transportation record from the Database:

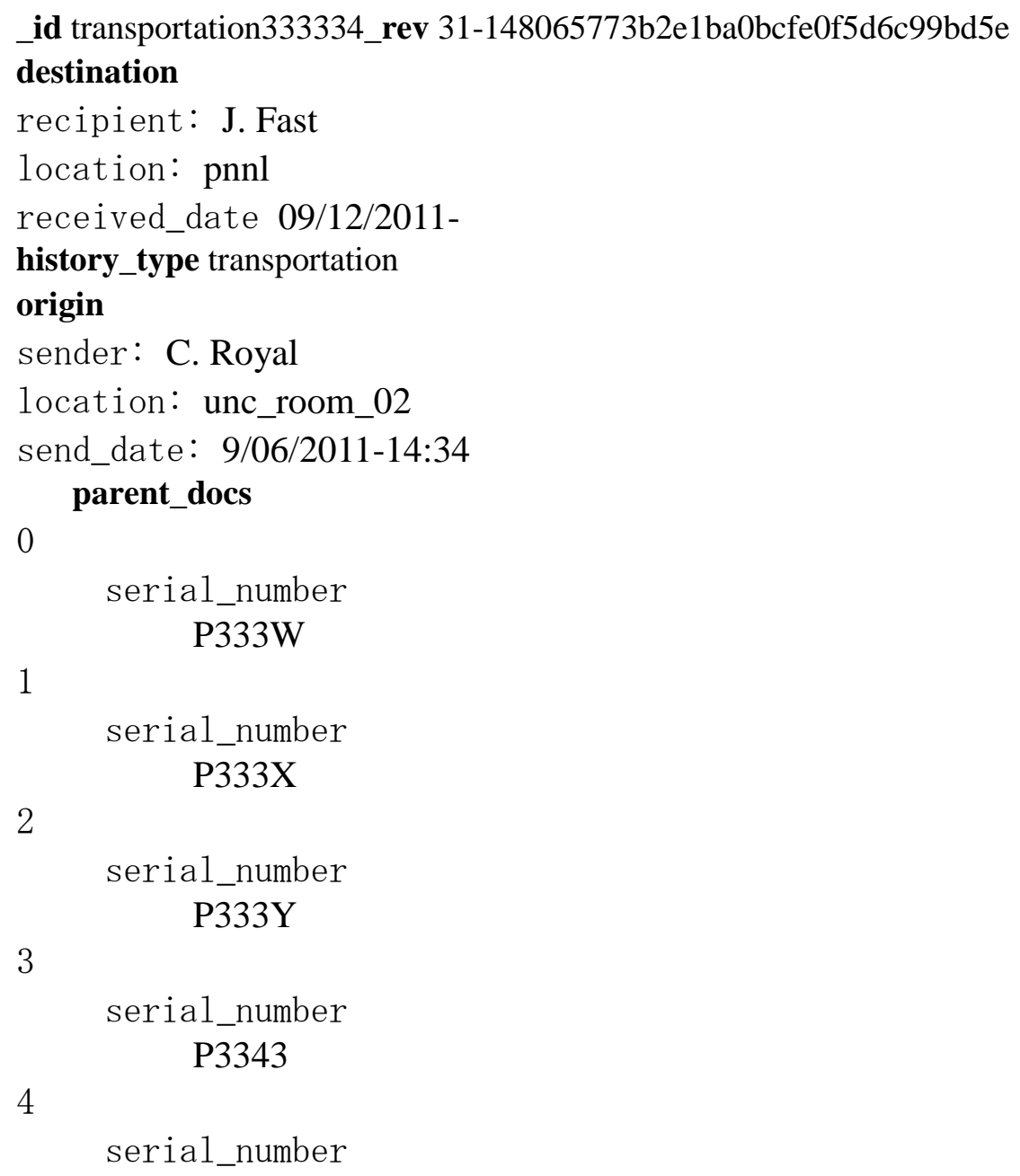


PNNL-21696

\section{P3344}

5

serial_number

P3346

record_type history_record

route_info FedEx - 1658259595

serial_number transportation333334

shipping_method Ground

status finished

transporter FedEx Freight

\subsection{Background estimation tool for the parts tracking database}

This estimation of equivalent sea level exposure during transportation relies on the known relationship between elevation and cosmic-ray flux - specifically since the cosmic ray flux increases exponentially at higher elevations. The tool finds the most likely route between the given locations using Google Maps, and samples the elevations along that route. It then integrates the exposure rate over that route to find the total. The equation for exposure rate used was:

$$
\text { Exposure Rate }=5^{\text {Altitude }} 1524 \mathrm{~m}
$$

This expression was taken from an excel spreadsheet that previously estimated exposure along routes. Due to the methods used, these are upper limits.

\begin{tabular}{|l|l|l|}
\hline From & To & $\begin{array}{l}\text { Exposure time (Hours sea level } \\
\text { equivalent) }\end{array}$ \\
\hline Rapid City, SD & PNNL, WA & 99.7 \\
\hline Denver, CO & Rapid City, SD & 32.6 \\
\hline Boise, ID & Denver, CO & 105.3 \\
\hline Hermiston, OR & Boise, ID & 12.4 \\
\hline Pasco, WA & Hermiston, OR & 1.0 \\
\hline PNNL, WA & Pasco, WA & 0.45 \\
\hline Rapid City, SD & Sanford, SD & 3.3 \\
\hline PNNL, WA & UNC, NC & 159.2 \\
\hline UNC, NC & LBNL, CA & 133.0 \\
\hline
\end{tabular}

Table 4: Sea-level equivalent exposure of different routes of interest for Majorana 


\subsection{Conclusions}

The problem of cosmogenic activation during transport of ultra-low background detector building materials is presented in this report. A muon monitor capable of aiding in the estimation of such contamination is described, and the process of doing such estimation from its measurements is detailed. The use of databases to log the routing of the different parts is also a tool that can help tremendously in tracking the activation of such parts. The muon monitor efficiency is calculated using Monte-Carlo simulations and the result is within 3\% from the measured efficiency of this detector. A software tool to estimate activation has been developed and will be used for activation monitoring in the MAJORANA DEMONSTRATOR experiment. 


\subsection{Acknowledgements}

The authors would like to thank Eric Hoppe for reviewing and providing valuable comments of this document and Jason Merriman for helping setting up the transport of the muon detector. The authors gratefully acknowledge the support of DOE-NP under grant \# DE-FG02-97ER41041, the NSF under grant \# PHY-0705014, and the State of North Carolina. 
PNNL-21696

\subsection{References}

Aguayo, E, JE Fast, RT Kouzes, JL Orrell. 2012. The $\mu$-Witness detector: A ruggedized, portable, flux meter for cosmogenic activation monitoring. [To be published.]

Aguayo, E, RT Kouzes, and JL Orrell. 2010. Monte Carlo Simulations of Cosmic Ray Hadronic Interactions. Report No. PNNL-20401, Pacific Northwest National Laboratory, Richland, Washington.

Rene Brun and Fons Rademakers, ROOT - An Object Oriented Data Analysis Framework, Proceedings AIHENP'96 Workshop, Lausanne, Sep. 1996, Nucl. Inst. \& Meth. in Phys. Res. A 389 (1997) 81-86. See also http://root.cern.ch/

Hagmann, C, D Lange, and D Wright. 2008. "Cosmic-Ray Shower Library (CRY)." Technical Manual, 5 pages. Lawrence Livermore National Laboratory, Livermore, CA. http://nuclear.llnl.gov/simulation/cry.pdf.

Gehman V. M,. Majorana Background Summary Document, 2008

Grieder PKF. 2001. Cosmic Rays at Earth. Elsevier.

Laubenstein, M and G Huesser. 2009. Cosmogenic radionuclides in metals as indicator for sea level exposure history. Applied Radiation and Isotopes 67, pp 750-754.

Ziegler, JF. 1998. Terrestrial cosmic ray intensities. IBM Journal of Research Development, Vol. 42, No. 1.

Knoll, GF. 2000. Radiation Detection and Measurement. 3rd ed., Wiley, New York. 
PNNL-21696

\section{Appendix A: Background estimation tool code}

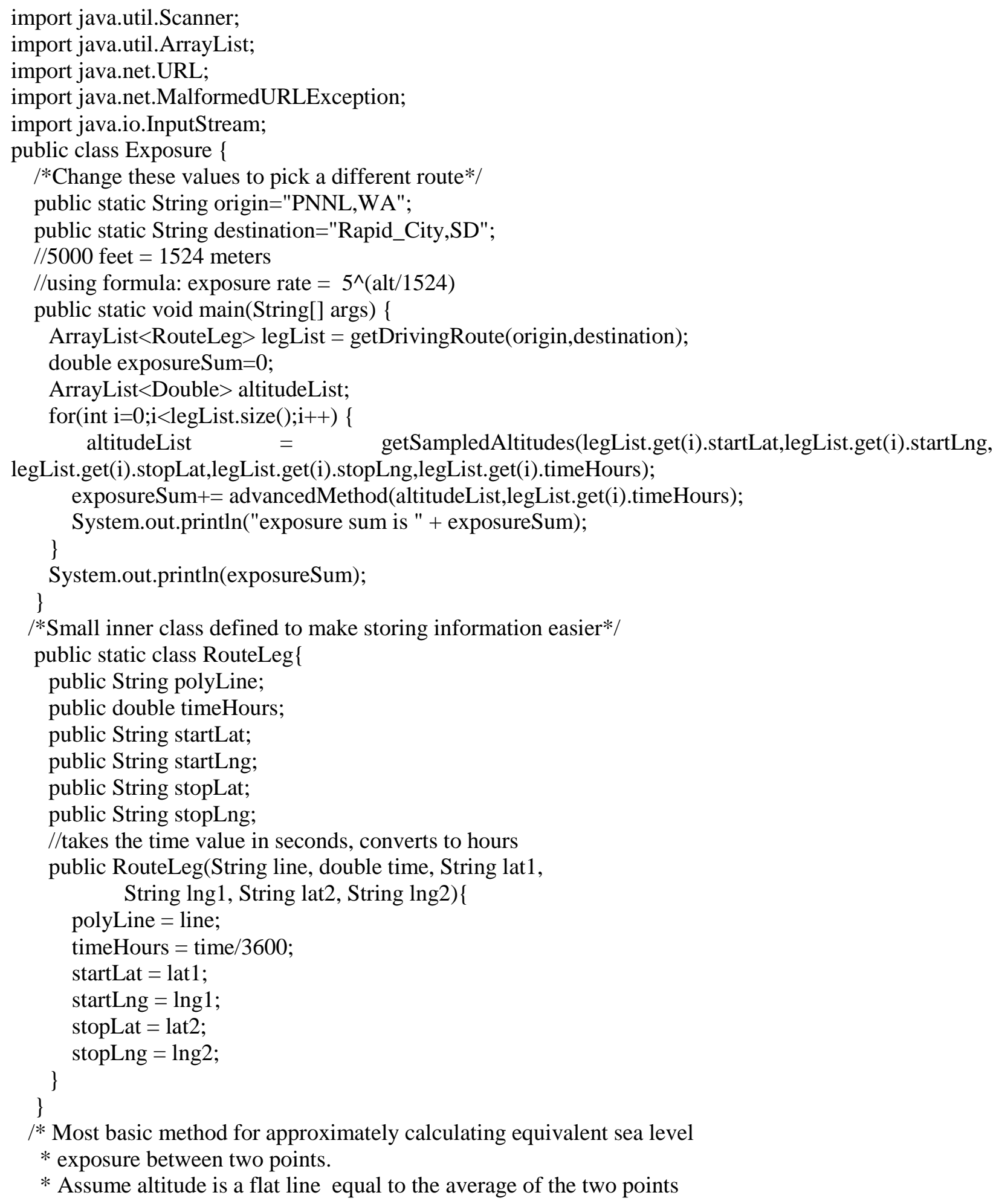

* Assume altitude is a flat line equal to the average of the two points 


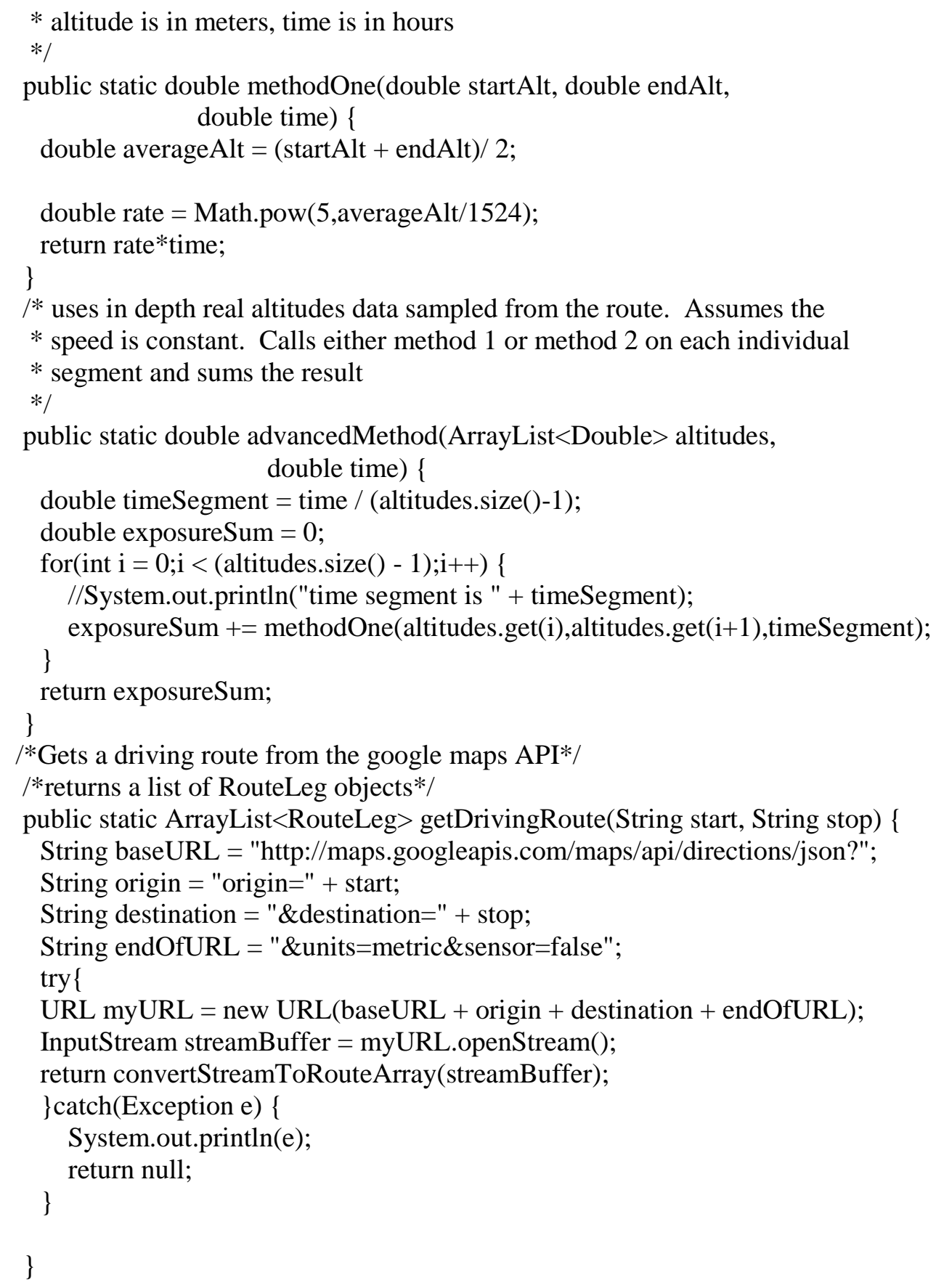


String lat $1=$ null, lat $2=$ null, $\operatorname{lng} 1=$ null, $\operatorname{lng} 2=$ =null;

double timeSeconds $=-1$;

Scanner stringScan = new Scanner(streamAsString);

ArrayList $<$ RouteLeg $>$ list $=$ new ArrayList $<$ RouteLeg $>()$;

/*skip forward past stuff we don't care about*/

while(stringScan.hasNext()) \{

if(stringScan.next().equals("\"steps\"")) \{

break;

\}

\}

/*parse the output*/

while(stringScan.hasNext()) \{

temp $=$ stringScan.next();

if(temp.equals("\"duration〉"")) \{

//get rid of useless data

stringScan.nextLine();

stringScan.nextLine();

stringScan.next();

stringScan.next();

//get the time in seconds

\}

timeSeconds $=$ stringScan.nextDouble();

else if(temp.equals("|"start_locationl"")) \{

stringScan.nextLine();

stringScan.next();

stringScan.next();

lat1 = stringScan.next () ;

//remove the comma

lat1 = lat1.substring(0,lat1.length()-1);

stringScan.nextLine();

stringScan.next();

stringScan.next();

$\operatorname{lng} 1$ = stringScan.next () ;

System.out.println(timeSeconds);

list.add(new RouteLeg(polyline,timeSeconds,lat1,lng1, lat2,lng2));

\}

else if(temp.equals("|"end_location〉"")) \{

stringScan.nextLine();

stringScan.next();

stringScan.next();

lat2 = stringScan.next () ;

lat2 = lat2 $\cdot \operatorname{substring}(0$, lat2.length()-1); 


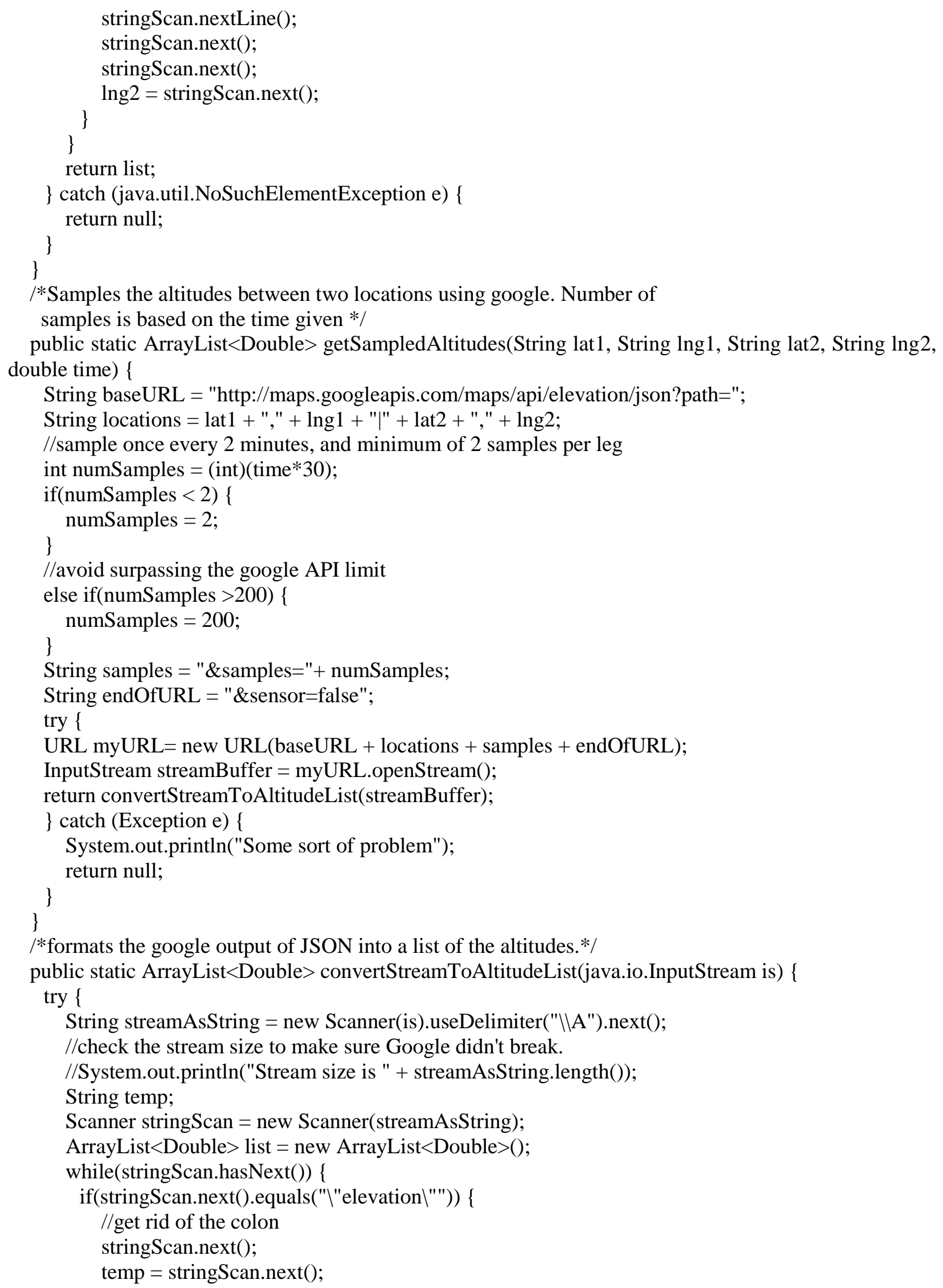


//remove the comma and add it to the list

list.add(new Double(temp.substring(0,temp.length()-1))); \}

\}

return list;

\} catch (java.util.NoSuchElementException e) \{ return null; \}

\} 


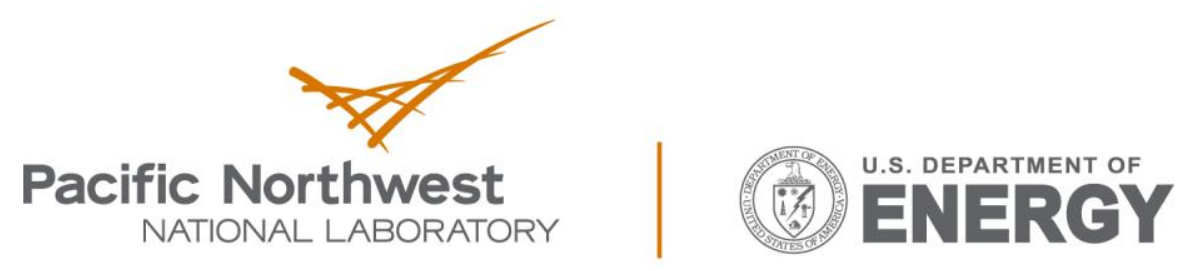

Proudly Operated by Battelle Since 1965

902 Battelle Boulevard

P.O. Box 999

Richland, WA 99352

1-888-375-PNNL (7665)

www.pnnl.gov 